Subscriber access provided by Uppsala universitetsbibliotek

\title{
Communication
}

\section{Two-electron-spin ratchets as a platform for microwave- free dynamic nuclear polarization of arbitrary material targets}

\author{
Pablo R. Zangara, Jacob Henshaw, Daniela Pagliero, Ashok Ajoy, \\ Jeffrey A. Reimer, Alexander Pines, and Carlos Andres Meriles \\ Nano Lett., Just Accepted Manuscript • DOI: 10.1021/acs.nanolett.8b05114 • Publication Date (Web): 18 Mar 2019 \\ Downloaded from http://pubs.acs.org on March 21, 2019
}

\section{Just Accepted}

"Just Accepted" manuscripts have been peer-reviewed and accepted for publication. They are posted online prior to technical editing, formatting for publication and author proofing. The American Chemical Society provides "Just Accepted" as a service to the research community to expedite the dissemination of scientific material as soon as possible after acceptance. "Just Accepted" manuscripts appear in full in PDF format accompanied by an HTML abstract. "Just Accepted" manuscripts have been fully peer reviewed, but should not be considered the official version of record. They are citable by the Digital Object Identifier (DOI®). "Just Accepted" is an optional service offered to authors. Therefore, the "Just Accepted" Web site may not include all articles that will be published in the journal. After a manuscript is technically edited and formatted, it will be removed from the "Just Accepted" Web site and published as an ASAP article. Note that technical editing may introduce minor changes to the manuscript text and/or graphics which could affect content, and all legal disclaimers and ethical guidelines that apply to the journal pertain. ACS cannot be held responsible for errors or consequences arising from the use of information contained in these "Just Accepted" manuscripts. 


\title{
Two-electron-spin ratchets as a platform for microwave-free dynamic nuclear polarization of arbitrary material targets
}

\author{
Pablo R. Zangara ${ }^{1}$, Jacob Henshaw ${ }^{1}$, Daniela Pagliero ${ }^{1}$, Ashok Ajoy ${ }^{3}$, Jeffrey A. Reimer ${ }^{4}$, Alexander Pines ${ }^{3}$, and Carlos \\ A. Meriles ${ }^{1,2 . \dagger}$ \\ ${ }^{1}$ Dept. of Physics, CUNY-City College of New York, New York, NY 10031, USA. \\ ${ }^{2}$ CUNY-Graduate Center, New York, NY 10016, USA. \\ ${ }^{3}$ Department of Chemistry, University of California Berkeley, and Materials Science Division Lawrence Berkeley National \\ Laboratory, Berkeley, California 94720, USA. \\ ${ }^{4}$ Department of Chemical and Biomolecular Engineering, and Materials Science Division Lawrence Berkeley National \\ Laboratory University of California, Berkeley, California 94720, USA.
}

\begin{abstract}
Optically-pumped color centers in semiconductor powders can potentially induce high levels of nuclear spin polarization in surrounding solids or fluids at or near ambient conditions, but complications stemming from the random orientation of the particles and the presence of unpolarized paramagnetic defects hinder the flow of polarization beyond the defect's host material. Here, we theoretically study the spin dynamics of interacting nitrogen-vacancy (NV) and substitutional nitrogen (P1) centers in diamond to show that outside protons spin-polarize efficiently upon a magnetic field sweep across the NV-P1 level anti-crossing. The process can be interpreted in terms of an NV-P1 spin ratchet, whose handedness - and hence the sign of the resulting nuclear polarization - depends on the relative timing of the optical excitation pulse. Further, we

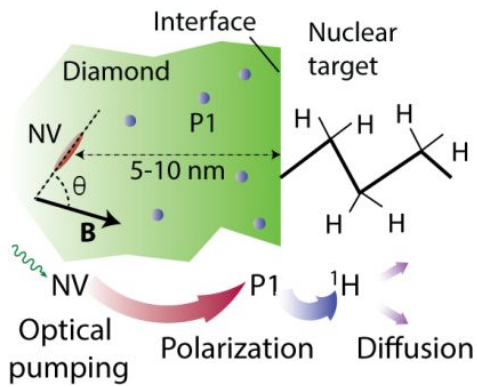
find that the polarization transfer mechanism is robust to $\mathrm{NV}$ misalignment relative to the external magnetic field, and efficient over a broad range of electron-electron and electron-nuclear spin couplings, even if proxy spins feature short coherence or spinlattice relaxation times. Therefore, these results pave the route towards the dynamic nuclear polarization of arbitrary spin targets brought in proximity with a diamond powder under ambient conditions.
\end{abstract}

KEYWORDS: Diamond, near-surface nitrogen-vacancy centers, P1 centers, dynamic nuclear polarization.

The creation of athermal nuclear spin states - i.e., whose absolute polarization is above that defined by Boltzmann statistics - is presently the center of a broad effort encompassing physics, chemistry, and materials science'. 'Solid-effect'-based schemes at high magnetic fields - involving microwave ( $\mathrm{mw}$ ) excitation of a radical embedded in a solid matrix - are presently prevalent, but their technical complexity (and corresponding cost) is driving a multi-pronged search for alternative pathways. Among the most promising routes is the use of color centers in insulators including, e.g., the negatively charged nitrogen-vacancy ${ }^{2-5}(\mathrm{NV})$ and other nitrogen-related ${ }^{6}$ centers in diamond, or the neutral di-vacancy center in silicon carbide ${ }^{7}$. Unlike other semiconductor-hosted paramagnetic defects (already exploited for dynamic nuclear polarization ${ }^{8,9}$ ), optical excitation spin-polarizes these color centers almost completely, even under ambient conditions. Therefore, rather than generating a relative polarization enhancement (crudely proportional to the operating magnetic field $\mathbf{B}$ and ratio $\mu_{\mathrm{e}} / \mu_{\mathrm{n}}$ between the electron and nuclear magnetic moments), opticallypumped color centers are capable of inducing high absolute nuclear spin Zeeman order at low fields ${ }^{2,10,11}$. Further, because of their comparatively long spin-lattice and coherence lifetimes, these spin-active color centers are amenable to electron/nuclear manipulation schemes difficult to implement in optically pumped organic molecules (where spin polarization builds up from shortlived excited triplets ${ }^{12}$ ).

While the larger surface-to-volume ratio makes the use of powdered semiconductors (as opposed to bulk crystals) better tailored to polarizing a surrounding matrix, the unavoidable misalignment between the applied magnetic field and the color center symmetry axis substantially complicates the transfer of magnetization ${ }^{13}$. In prior work, we demonstrated that ${ }^{13} \mathrm{C}$ spins in NV-hosting diamond particles can be efficiently polarized through the combined use of continuous optical excitation and mw frequency sweeps ${ }^{14,15}$. To reach nuclear spins outside the diamond lattice, however, polarization must spin diffuse from carbons adjacent to the $\mathrm{NV}$, a relatively slow process that can be hampered by the presence of other unpolarized paramagnetic defects.

Here we theoretically explore an alternate, mw-free approach where shallow paramagnetic defects operate as proxy spins to mediate the transfer of polarization from a source color center deeper in the host lattice to outside nuclei. For concreteness, we consider the case of NV centers and substitutional nitrogen in diamond - the so- 
(a)

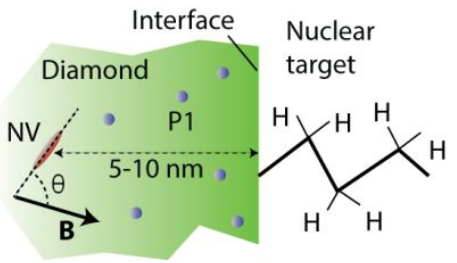

(b)

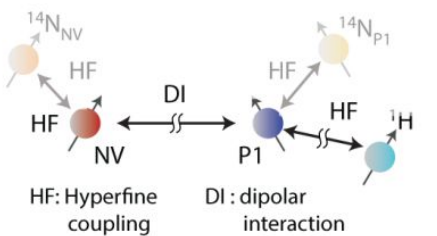

(c)

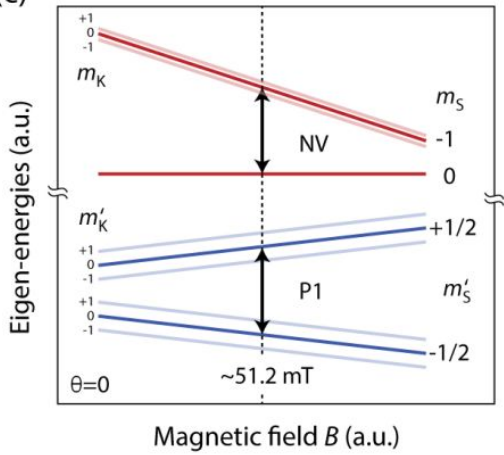

(d) Magnetic field $B(\mathrm{mT})$
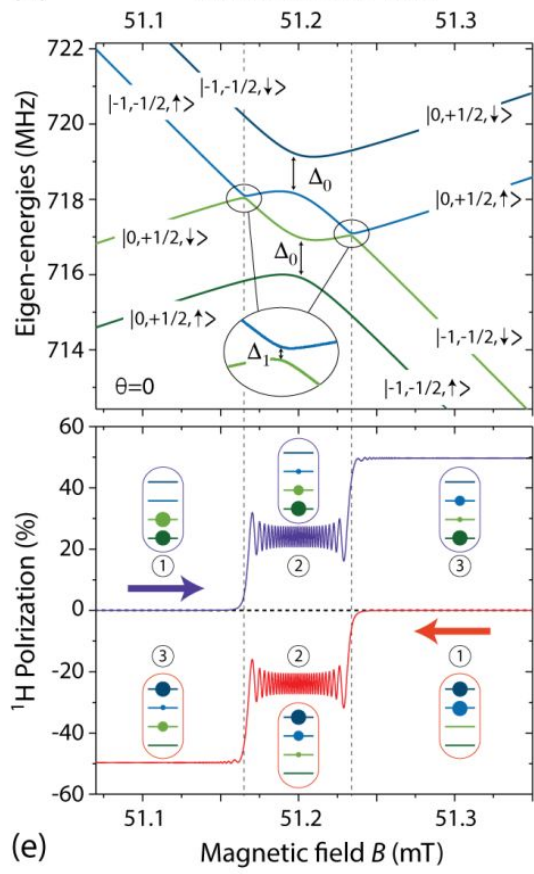

(f)

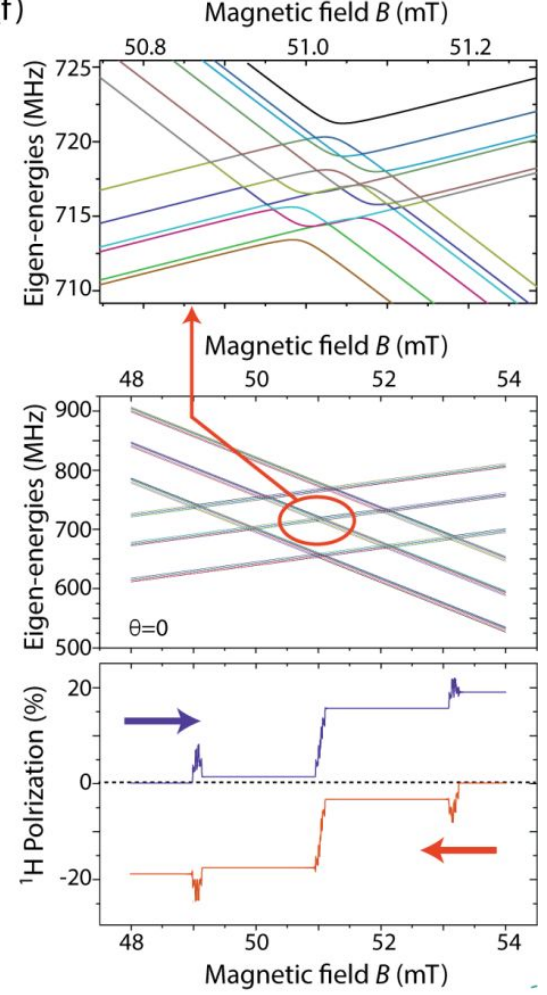

Figure 1. Proxy-spin-mediated polarization transfer to weakly-coupled proton spins. (a) Typically, the NV and target outer nuclear spins (protons in this case) are separated by at least 5-10 nm, thus leading to comparatively weaker couplings with protons on the surface; more proximal P1 centers (and other spin-1/2 surface defects) can thus serve as proxies to mediate the polarization transfer. (b) Electron/nuclear spin model; unless explicitly noted, we ignore the NV and P1 hyperfine interactions with their ${ }^{14} \mathrm{~N}$ hosts (faded spins). (c) Schematic energy diagram for the NV and P1 centers; near $51 \mathrm{mT}$, the NV $\left|m_{\mathrm{S}}=0\right\rangle \leftrightarrow$ $\left|m_{\mathrm{S}}=-1\right\rangle$ and P1 $\left|m_{\mathrm{S}}^{\prime}=+1 / 2\right\rangle \leftrightarrow\left|m_{\mathrm{S}}^{\prime}=-1 / 2\right\rangle$ transitions are nearly matched. (d) Level anti-crossing near $51 \mathrm{mT}$ for the combined NV-P1-1 $\mathrm{H}$ spin system without considering the hyperfine couplings with the ${ }^{14} \mathrm{~N}$ spins. (e) Calculated ${ }^{1} \mathrm{H}$ spin polarization as we sweep the magnetic field from left to right or from right to left (purple and red trances, respectively). Inserts indicate relative populations using the color code in (d). (f) Same as in (d,e) but taking into account the NV and P1 hyperfine couplings with their respective ${ }^{14} \mathrm{~N}$ hosts; the upper plot is a blown out view of the energy level structure within the circled region. Throughout these calculations, we assume the NV axis and external magnetic field are parallel $(\theta=0)$, the NV-P1 coupling is $500 \mathrm{kHz}$, the $\mathrm{P} 1{ }^{1} \mathrm{H}$ coupling is $200 \mathrm{kHz}$, and the field sweep rate is $0.26 \mathrm{mT} / \mathrm{ms}$; we also assume optical excitation pumps the NV spin to $100 \%$ and all other spin species are unpolarized.

called 'P1 center' - but the ideas we lay out can be naturally extended to other spin systems. Our approach exploits the 'cross effect', where nuclear spins polarize thanks to the slight energy mismatch between the source and proxy spins ${ }^{16-18}$. Unlike traditional, mw-mediated implementations, however, here we show that protons proximal to $\mathrm{P} 1$ centers can be polarized efficiently through protocols articulating pulsed optical excitation and low magnetic field sweeps. Remarkably, we attain additive dynamic nuclear polarization during both the 'low-tohigh' and 'high-to-low' segments of the field sweep cycle, with the sign of the nuclear polarization depending on the relative timing of the optical excitation pulse. Further, we show that proxy spins featuring short coherence or spinlattice relaxation times can efficiently contribute to the process, and that the technique is robust over a broad set of inter-spin couplings and relative orientations of the NV axis and $\mathbf{B}$ field. For completeness, we note that related studies involving optically-initialized color centers and proxy spins have been discussed in the context of nanoscale quantum sensing ${ }^{19-21}$

Fig. 1a sketches the starting nanoscale geometry: NV centers - in general, arbitrarily oriented relative to $\mathbf{B}-$ coexist with more abundant P1 centers and other shallow paramagnetic defects (e.g., dangling bonds at the diamond surface, not shown). Band bending effects - prone to remove the excess electron from the negatively charged $\mathrm{NV}$ - impose a minimum distance to the surface ${ }^{22}$ (at best of order 5-10 nm), meaning that the NV interaction with outside nuclear spins (protons in the case considered herein) is comparatively weak. Paramagnetic centers in the region separating the $\mathrm{NV}$ from the nuclear target are ideally positioned to mediate the transfer of polarization, but their faster transverse and spin-lattice relaxation as well as the broad distribution of coupling strengths between the source, proxy, and target spins poses a number 
of difficulties seemingly difficult to overcome.

To quantitatively model our polarization scheme, we consider the spin cluster in Fig. 1b comprising an NV coupled to a P1 center (a spin-1/2 defect); the latter also interacts with a proton spin on the diamond surface via a dipolar-type hyperfine coupling. Both the NV and P1 electron spins are hyperfine coupled to the nuclear spins of their respective nitrogen hosts (typically taking the form of ${ }^{14} \mathrm{~N}$ isotopes). We later show, however, these interactions do not significantly impact the polarization flow to the proton, and can thus be ignored (see Hamiltonian formulation in the Supporting Information, Section S.I). For clarity, we first consider the energy diagram for the NV-P1 pair in the case where the NV symmetry axis and magnetic field are collinear $(\theta=0)$. Near $51 \mathrm{mT}$, the P1 Zeeman splitting matches the energy separation corresponding to the $\left|m_{S}=0\right\rangle \leftrightarrow\left|m_{S}=-1\right\rangle$ transition of the NV, thus leading to cross-relaxation between both electron spins ${ }^{23}$. Slightly above and below the matching field, energy conservation in the electron-electron spin transition can be regained with the assistance of a coupled nuclear spin, which flips in one direction or the other depending on the sign of the difference between the NV and P1 splittings. Since continuous optical illumination spin pumps the NV into $\left|m_{\mathrm{S}}=0\right\rangle$, cross relaxation leads to $\mathrm{P} 1$-assisted dynamic nuclear polarization, whose sign alternating from positive to negative - depends on the chosen magnetic field ${ }^{17,18}$.

The route we pursue herein starts with the use of an optical pulse to initialize the NV spin into $\left|m_{S}=0\right\rangle$, followed by a gradual change of the magnetic field amplitude $B$ across the range where electron/nuclear cross relaxation takes place. To understand the ensuing spin dynamics, we first plot the energy diagram for the NV-P1${ }^{1} \mathrm{H}$ set as a function of $B$ in the simplified case where the $\mathrm{NV}$ and $\mathrm{P} 1$ hyperfine interactions with the nuclear spins of their respective nitrogen hosts is zero (Fig. 1d). We find a series of avoided crossings with energy gaps strongly dependent on the particular pair of eigenstates. In our calculations, we choose the field sweep rate so as to make the Landau Zener (LZ) dynamics partially non-adiabatic at the narrower gaps between the two inner branches (featuring opposite nuclear spin numbers), i.e., we set $\beta \equiv$ $d B / d t \quad$ so that $p_{1}>p_{0} \sim 0$, where $p_{i} \sim \exp$ $\left\{-\pi \Delta_{i}^{2} /\left(4\left|\gamma_{e}\right| \beta\right)\right\}, \quad i=0,1 \quad$ is the Landau-Zener probability of bifurcation, $\gamma_{e}$ is the electron gyromagnetic ratio, and $\Delta_{1}\left(\Delta_{0}\right)$ denotes the narrower (wider) energy gap (Fig. 1d, see analytical estimates for $\Delta_{1}$ and $\Delta_{0}$ in the Supporting Information, Section S.II). Therefore, assuming no initial ${ }^{1} \mathrm{H}$ magnetization (stage (1) in Fig. 1e), sweep-induced population exchange during the narrower LZ crossings leads to the generation of net nuclear spin polarization (stages (2) and (3)), as confirmed by direct numerical computations both for low-to-high and high-to- low field sweeps (upper and lower plots in Fig. 1e). Note that proton polarization of the opposite sign builds up in one case or the other; this behavior is somewhat reminiscent of that experimentally observed for carbons in NV-hosting diamonds simultaneously subjected to $\mathrm{mw}$ frequency sweeps and continuous optical illumination ${ }^{14}$. We later show, however, it is the relative timing of the illumination rather than the sweep direction what defines the sign of the resulting nuclear polarization.

An accurate description of the system spin dynamics must take into account the NV and P1 hyperfine couplings with their nuclear spin hosts. Assuming ${ }^{14} \mathrm{~N}$ isotopes in both cases - featuring spin numbers $K, K^{\prime}=1$ - each level in the energy diagram of Fig. 1e splits into nine distinct branches, corresponding to different combinations of the quantum projections $m_{\mathrm{K}}, m_{\mathrm{K}}^{\prime}=0, \pm 1$. Given the dominant character of the $\mathrm{P} 1-{ }^{14} \mathrm{~N}$ hyperfine coupling (of order $\sim 100 \mathrm{MHz}$ ), the diagram shows well-resolved eigenenergy sets (center plot in Fig. 1f), but the high multiplicity leads to subtle structures at the avoided crossings (upper insert in Fig. 1f). Despite this complexity, the system response upon a field sweep retains the main traits found in the simpler case (i.e., Fig. 1d), namely, the proton spin polarizes efficiently with a sign dependent on the sweep direction (lower graph in Fig. 1f). Similar to Fig. 1e, we attain near-optimal levels of proton polarization, though quantum interferences during the LZ crossings make the exact value a sensitive function of the sweep rate (see Supporting Information, Section S.III). To gain physical intuition (and speed up computations), we henceforth ignore the hyperfine coupling with the nitrogen hosts with the understanding that these contributions may only slightly alter some of the numerical values we derive, without fundamentally modifying the underlying transfer processes.

Since the spin dynamics is insensitive to the exact start and end magnetic field values, the results in Fig. 1 indicate that P1-assisted DNP can be made robust to field heterogeneities (and, as we show later, to spin coupling dispersion and $\mathrm{NV}$ orientation disorder). On the other hand, the slow sweep rate required for optimal efficiency $(\sim 0.3 \mathrm{mT} / \mathrm{ms})$ is at odds with the relatively short coherence and spin-lattice relaxation times of both electron spins near the diamond surface. Further, because unlike mw, a magnetic field must be present at all times, the impact of a full field cycle (including the low-to-high and high-to-low ramps) on the end proton polarization is a priori unclear.

We address these issues in Fig. 2a, where we monitor the nuclear spin evolution as we complete successive field cycles using a tenfold faster sweep rate $(3 \mathrm{mT} / \mathrm{ms})$. Somewhat unexpectedly, we find that nuclear polarization adds constructively during both halves of the field cycle, with the sign being determined by the illumination timing rather than the sweep direction: Positive (negative) ${ }^{1} \mathrm{H}$ polarization emerges from NV spin initialization at the low 
(a)

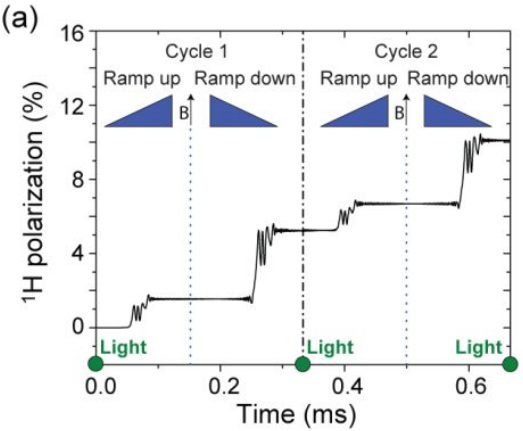

(d)

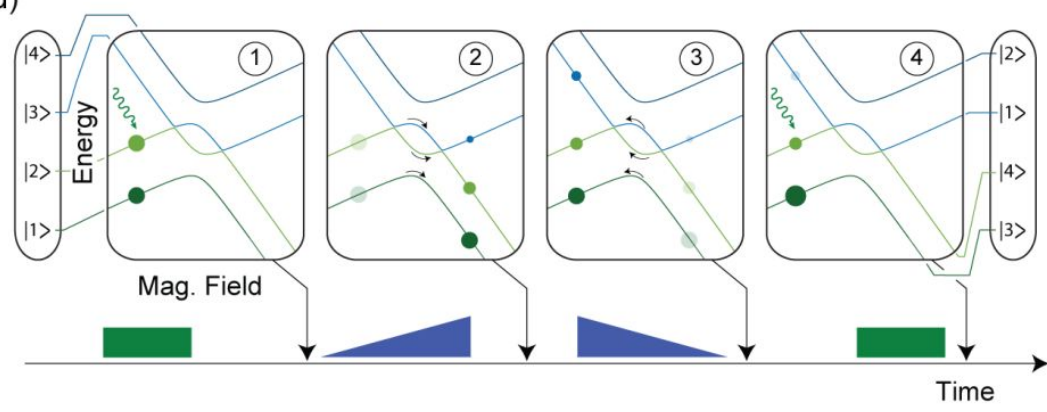

(b)

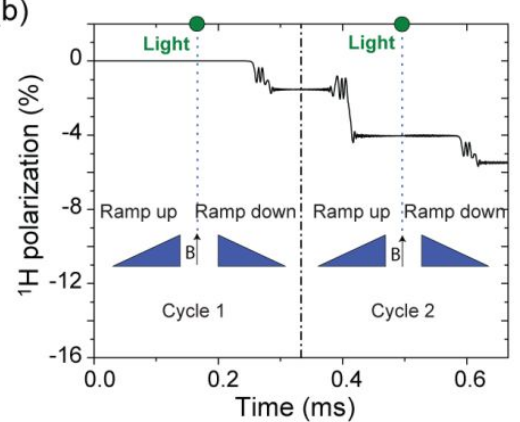

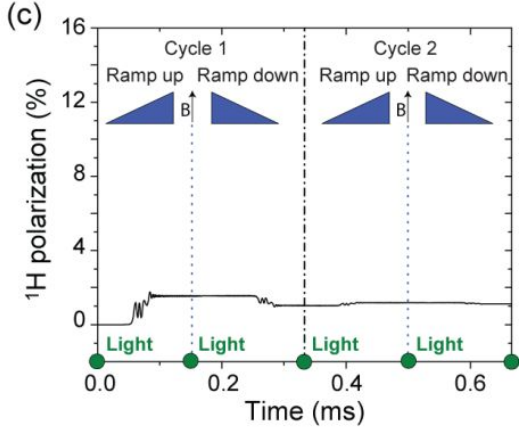

(e)

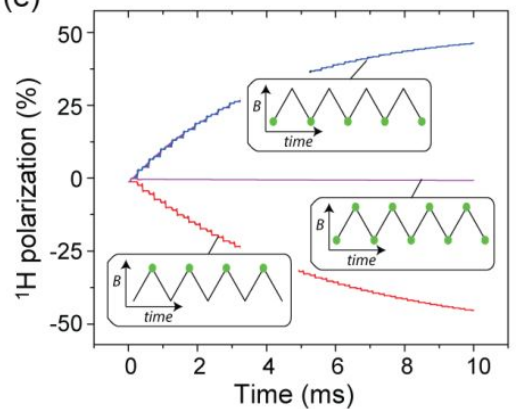

Figure 2. The two-electron spin ratchet. (a) Proton dynamic polarization upon two full magnetic field cycles; the field range is centered around the NV-P1- 1 H level anti-crossing at $\sim 51 \mathrm{mT}$ and $\mathrm{NV}$ spin preparation takes place at the low field point. (b) Same as in (a) but assuming optical excitation at the time when the magnetic field is at its maximum. (c) Same as in (a) but for a sequence where NV spin pumping takes place both at the beginning and midpoint of each cycle. (d) Schematics of the proton polarization buildup upon application of the protocol in (a). Green squares (blue triangles) indicate laser pulses (magnetic field ramps). Rounded squares in the upper half reproduce the energy diagram from Fig. 1a. Spin populations are represented by solid circles of variable radius; for clarity, we assume the laser pulse fully projects the NV spin into $m_{\mathrm{S}}=0$, though only partial spin pumping is required. Here we use the state notation $|1\rangle=|0,+1 / 2, \uparrow\rangle,|2\rangle=|0,+1 / 2, \downarrow\rangle,|3\rangle=$ $|-1,-1 / 2, \uparrow\rangle,|4\rangle=|-1,-1 / 2, \downarrow\rangle$, with labels representing the NV, P1, and ${ }^{1} \mathrm{H}$ quantum projections, respectively. (e) Evolution of the ${ }^{1} \mathrm{H}$ spin polarization as a function of time upon multiple repetitions of the optical excitation/field sweep cycles described above. Throughout these calculations, $\theta=0$, we use $\dot{v}_{\mathrm{B}}=3 \mathrm{mT} / \mathrm{ms}$, and the field range is $0.5 \mathrm{mT}$. Further, the NV-P1 and $\mathrm{P} 1-{ }^{1} \mathrm{H}$ couplings are respectively $500 \mathrm{kHz}$ and $100 \mathrm{kHz}$, and the $\mathrm{NV}$ spin polarization upon optical excitation is $100 \%$; we consider no transverse or longitudinal relaxation processes.

field (high-field) extremum of the cycle (Figs. 2a and 2b, respectively). Consistent with this response, we observe negligible DNP for a cycle with optical illumination at both extrema (Fig. 2c), with the imperfect cancellation between the low-to-high and high-to-low halves arising from the slight asymmetry in the initial spin populations at each half period during the first few repeats (see Supporting Information, Sections S.IV).

The formal description of the process is not simple, but can be attained with the use of a transfer matrix (TM) model (see Supporting Information, Section S.V). To qualitatively illustrate the underlying dynamics, Fig. 2d follows the evolution of the proton spin polarization throughout a cycle of magnetic field sweeps and optical excitation at the low-field-end of the ramp (the protocol in Fig. 2a); for simplicity, we assume that laser excitation fully spin pumps the NV into $m_{\mathrm{S}}=0$, though we note that only a partial spin projection is required. In the regime of moderately fast field sweeps where $p_{1} \lesssim 1$ and $p_{0} \sim 0$, both inner branches nearly exchange their populations during each of the two LZ crossings, while the populations of the outer branches remain unchanged. Correspondingly, only a small (positive) nuclear spin imbalance emerges from the low-to-high field ramp (stage (2) in Fig. 2d). As a simple visual inspection shows, however, this difference virtually doubles if one subsequently forces the system to undergo a reversed, high-to-low field ramp (stage (3) in Fig. 2d). Subsequent optical excitation - acting exclusively on the $\mathrm{NV}$ - leaves the nuclear spin polarization unchanged and thus resets the system for a new DNP cycle (stage (4) in Fig. 2d).

Repeated application of the same protocol leads to gradual accumulation of proton polarization in the form of an exponential growth towards a near-optimum value approaching the starting NV spin polarization (Fig. 2d). This one-directional flow of spin polarization upon a timeperiodic, zero-mean modulation of the magnetic field is analogous to the directed motion of quantum motors ${ }^{24}$, prompting us to interpret the dynamics as that of a twoelectron spin ratchet. Note that in the absence of transverse or spin lattice relaxation - the limit assumed so far, see below - the time interval between successive optical pulses can be increased so as to encompass multiple field cycles $l>1$. The corresponding response - in general, a 

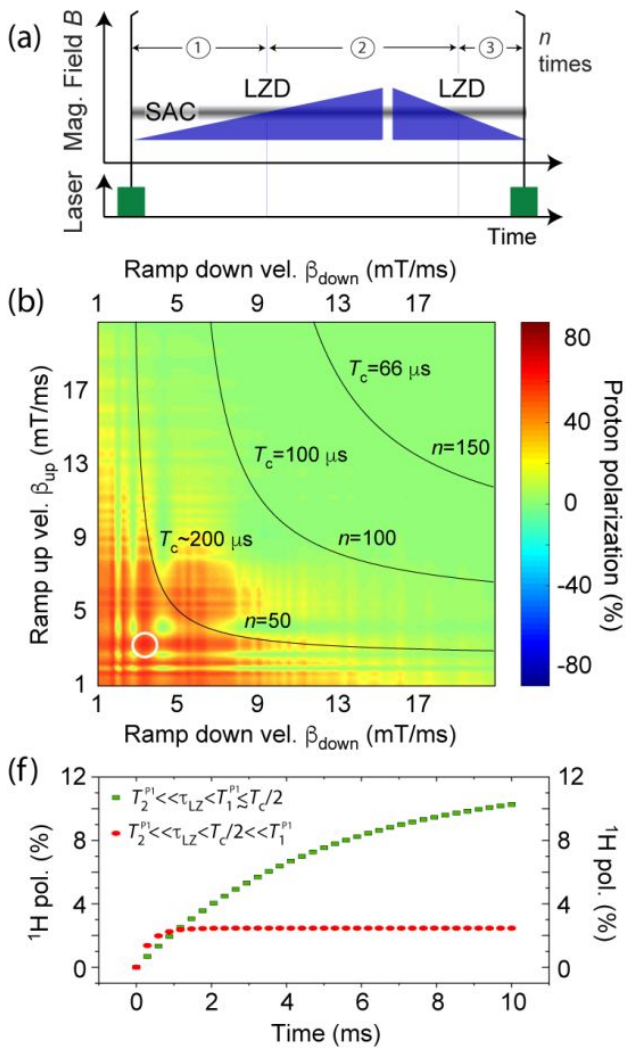

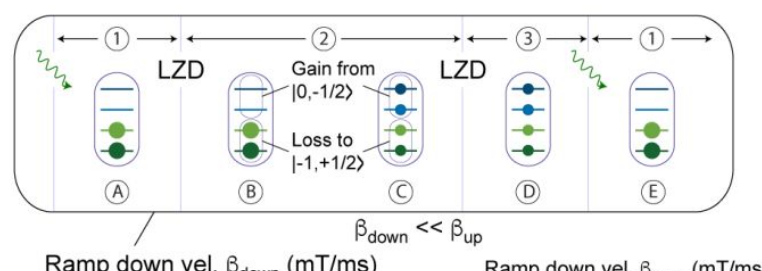

$\begin{array}{llllll}\text { (c) } & 1 & 5 & 9 & 13 & 17\end{array}$

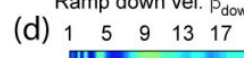
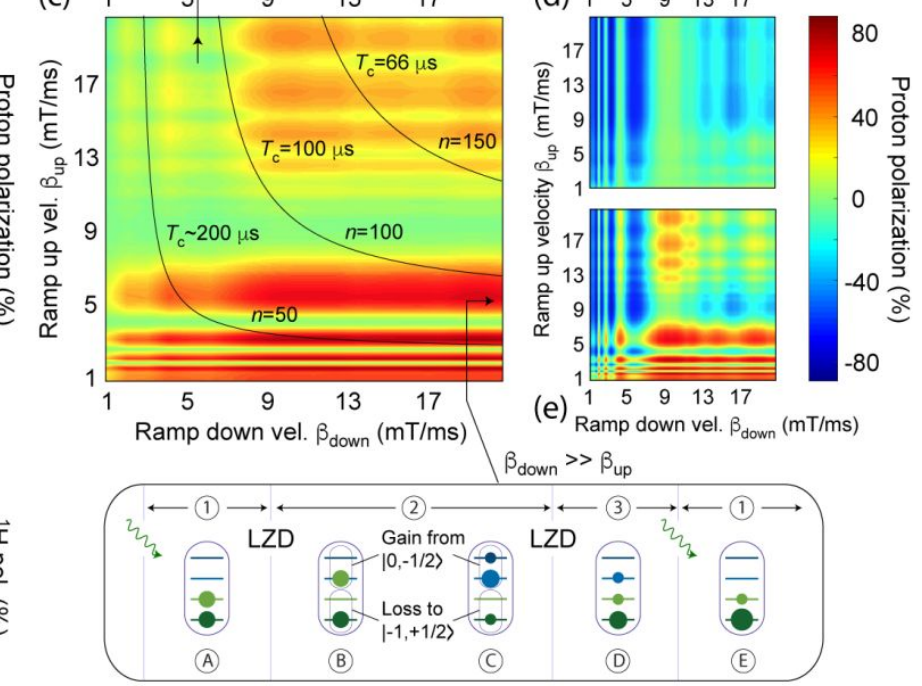

Figure 3. Proxy-spin-mediated DNP in the presence of spin-lattice relaxation. (a) Generalized DNP protocol featuring variable low-to-high and high-to-low field sweep velocities. (b) Calculated ${ }^{1} \mathrm{H}$ spin polarization as a function of the 'ramp up' and 'ramp down' velocities for a total DNP time of $10 \mathrm{~ms}$ in the regime $\tau_{\mathrm{LZ}}<T_{2}^{(\mathrm{P} 1)} \leq T_{\mathrm{c}} / 2 \ll T_{1}^{(\mathrm{P} 1)}$; optical excitation takes place at the low-field end of the ramp. Solid lines indicate areas of the plot sharing the same cycle time $T_{\mathrm{c}}$, and hence undergoing the same number $n$ of DNP repeats. (c) Same as in (b) but assuming the $\mathrm{P} 1$ spin relaxes before and after traversing the set of avoided crossings so that $\tau_{\mathrm{LZ}}<T_{2}^{(\mathrm{P} 1)} \leq T_{\mathrm{c}} / 2 \sim T_{1}^{(\mathrm{P} 1)}$. The upper and lower inserts highlight the impact of P1 spin-lattice relaxation throughout the DNP cycle in (a) for the limit cases where one field ramp is much faster than the other one; for simplicity, we collapse the NV-P1- ${ }^{1} \mathrm{H}$ energy diagrams to sets of four horizontal lines, each corresponding to the branch in Fig. $1 \mathrm{~d}$ with the same color code. (d) Same as in (c) but for optical excitation at the high-field end of the ramp. (e) Same as in (c) but assuming optical excitation both at the low- and high-field ends of the ramp. (f) Proton spin polarization buildup as a function of time upon repeated applications of the DNP protocol when $T_{2}^{(\mathrm{P} 1)} \ll \tau_{\mathrm{LZ}}<T_{\mathrm{c}} / 2 \ll T_{1}^{(\mathrm{P} 1)}$ (red ellipses) or when $T_{2}^{(\mathrm{P} 1)} \ll \tau_{\mathrm{LZ}}<T_{\mathrm{c}} / 2 \sim T_{1}^{\mathrm{P} 1)}$ (green rectangles). In both cases, we assume $\beta_{\text {down }}=\beta_{\text {up }}=3 \mathrm{mT} / \mathrm{ms}$ and $T_{2}^{\mathrm{P} 1)}=100 \mathrm{~ns}$. In all plots, we assume $\theta=0$, the field range is $0.5 \mathrm{mT}$, the NV-P1 and $\mathrm{P} 1-{ }^{1} \mathrm{H}$ couplings are respectively $500 \mathrm{kHz}$ and $100 \mathrm{kHz}$, and the NV spin polarization upon optical excitation is $100 \%$. In (a) and (c), SAC denotes the 'set of avoided crossings' approximately midway within the magnetic field range and LZD indicates Landau-Zener dynamics.

function of $l$ - remains comparable to that shown in Fig. $2 \mathrm{e}$, both in terms of the nuclear polarization buildup rate and limit value, though we warn that quantum interference effects between successive passages can make the growth strongly non-monotonic (see Supporting Information, Section S.IV).

Naturally, the field sweep cycle of Fig. 2 can be generalized to the case where the low-to-high and high-tolow field sweep rates (respectively denoted $\beta_{\text {up }}$ and $\beta_{\text {down }}$ ) are unequal (Fig. 3a). Fig. 3b shows the calculated proton polarization for periodic illumination at each low-field extremum assuming the total DNP time remains fixed (10 $\mathrm{ms}$ ). To avoid quantum interferences between successive crossings, we impose the condition $\tau_{\mathrm{LZ}}<T_{2}^{(\mathrm{P} 1)}<T_{\mathrm{c}}$, where $T_{2}^{(\mathrm{P} 1)}$ denotes the $\mathrm{P} 1$ transverse coherence lifetime (assumed shorter than $\left.T_{2}^{(\mathrm{NV})}\right), T_{\mathrm{c}}$ is the polarization cycle period, and $\tau_{\mathrm{LZ}} \sim \Delta_{0} /\left(2\left|\gamma_{\mathrm{e}}\right| \beta\right)$ is the characteristic LandauZener time ${ }^{25}$, required for a coherent transfer (see below). The interplay between polarization transfer efficiency and multiple repetitions leads to near optimal proton polarization over a broad set of up/down sweep rate combinations, though optimal DNP is restricted to the lower left corner of the plot, corresponding to DNP cycle times $T_{\mathrm{c}} \gtrsim 0.2 \mathrm{~ms}$.

We are now in a position to investigate the influence of spin relaxation, which, as we show next, can have a profound effect on the system response. For simplicity, we assume that only the P1 spin relaxes during a DNP cycle (i.e., $T_{1}^{(\mathrm{P} 1)}<T_{1}^{(\mathrm{NV})}$ ), a condition justified in the present case given the imposed P1 proximity to the diamond surface (and/or spin exchange with other paramagnetic defects in its neighborhood). Fig. 3c shows the result of a 
calculation where we impose $T_{1}^{(\mathrm{P} 1)}=T_{\mathrm{c}} / 2$ so as to force $\mathrm{P} 1$ spin relaxation after the first (but before the second) LZ pass in a cycle across the set of avoided crossings (SAC). Unlike Fig. $3 b$ - insensitive to an exchange of the up and down field sweep rates - the presence of a finite $T_{1}^{(\mathrm{P} 1)}$ time introduces a strong asymmetry (Fig. 3c). Optimal nuclear polarization builds up for arbitrarily large $\beta_{\text {down }}$ rates so long as $\beta_{\text {up }}$ does not exceed an upper threshold ( 7 $\mathrm{mT} / \mathrm{ms}$ for the present set of couplings), but the converse is not true.

The impact of spin relaxation, though complex, can be formally incorporated in our TM approach (see Supplementary Information, Section VI). A simpler, more intuitive understanding of the underlying dynamics, however, can be gained by considering the evolution of spin populations in the regime where the faster passage in a non-symmetric field cycle is fully non-adiabatic (i.e., when $p_{0} \sim p_{1} \sim 1$, upper and lower inserts in Fig. 3c). If the first half of the cycle is the faster one, spin-lattice relaxation before traversing the set of avoided crossing a second time populates all four energy branches equally, meaning that the ensuing LZ dynamics cannot produce nuclear polarization regardless the sweep rate (upper insert in Fig. 3c). The result is different in the converse regime (i.e., when the second sweep is faster), because P1 spinlattice relaxation does not degrade the nuclear spin population created during the first pass (lower insert in Fig. $3 c$, and Supplementary Information, Section S.IV). In other words, positive nuclear spin polarization can be produced in the limit where $\beta_{\text {down }} \gg \beta_{\text {up }}$ but the inverse is not true. Interestingly, spin-lattice relaxation can still induce substantial nuclear polarization even when both sweep rates are comparably fast (upper right corner in the main plot of Fig. 3c), through the underlying dynamics is more complex (Supplemental Information, Sections S.IV through S.VI).

For completeness, we note that NV spin initialization at the high-field extremum of the cycle (i.e., the generalization of the protocol in Fig. 2b) simply produces a reversal in the asymmetry, i.e., negative nuclear polarization emerges for $\beta_{\text {down }} \ll \beta_{\text {up }}$ (Fig. 3d). Likewise, optical excitation both at the low- and high-field extrema can yield net nuclear polarization of one sign or the other whenever $\beta_{\text {down }} \neq \beta_{\text {up }} \quad$ (Fig. 3e). As a corollary, continuous laser illumination (as opposed to synchronous, pulsed optical excitation) should yield efficient DNP provided the two sweep rates are substantially different from each other.

Given the short spin lifetimes typical in near-surface paramagnetic centers, the regime $T_{2}^{(\mathrm{P} 1)}<\tau_{\mathrm{LZ}}-$ corresponding to the strongly dissipative limit - deserves special consideration. In this regime, coherent transfer of the spin polarization is not possible and the system dynamics is better described via the Landau-Zener formulas for the case of strong-dephasing26-28 (see Supplementary Information, Sections V and VI). The impact of fast P1 decoherence is illustrated in Fig. $3 \mathrm{f}$ assuming $T_{2}^{(\mathrm{P} 1)}=100 \mathrm{~ns}$ and $\beta_{\text {down }}=\beta_{\text {up }}=3 \mathrm{mT} / \mathrm{ms}$ (white circle on the plot of Fig. 3b): Under these extreme conditions, $T_{2}^{(\mathrm{P} 1)} \ll \tau_{\mathrm{LZ}} \sim 5 \mu \mathrm{s}$ and, in the limit $T_{\mathrm{c}} \ll T_{1}^{(\mathrm{P} 1)}$, we calculate strongly attenuated proton polarization buildup (red circles in Fig. 3f). However, for shorter P1 spin lifetimes $\tau_{\mathrm{LZ}}<T_{1}^{(\mathrm{P} 1)} \lesssim T_{\mathrm{c}} / 2$, we find that a substantial fraction of the original DNP efficiency can be regained (i.e., P1 spin lattice relaxation partially remedies fast decoherence, green dots in Fig. 3f).

Since in a realistic setting the spatial separations between the source, proxy, and target spins change randomly, efficient polarization transfer to outside nuclei requires the DNP protocol to be robust to spin coupling heterogeneity. We address this issue in Fig. 4a, where we calculate the proton polarization upon application of the sequence in Fig. 2a as a function of both the NV-P1 and $\mathrm{P} 1-{ }^{1} \mathrm{H}$ couplings. Remarkably, we attain near optimal polarization transfer over a broad set of conditions extending down to NV-P1 $\left(\mathrm{P}^{-}{ }^{-} \mathrm{H}\right)$ couplings as weak as $\sim 300 \mathrm{kHz}(\sim 200 \mathrm{kHz})$. The latter corresponds to sourceproxy (proxy-target) spin separations as large as $\sim 5 \mathrm{~nm}(\sim 1$ $\mathrm{nm}$ ). These distances are typical in samples that have been engineered to host shallow $\mathrm{NVs}^{29}$, and hence compatible with proxy-spin-mediated polarization transfer to outside nuclear targets (see lower insert in Fig. 4a).

Finally, we investigate the DNP efficiency as a function of the magnetic field orientation relative to the three-spin set. For our calculations, we choose a reference frame whose $z$-axis points along the NV direction, and where the $x z$-plane matches that defined by the NV and the NV-P1 axes (Fig. 4b). In order to study the LZ dynamics away from $\theta=0$, we first consider an isolated NV-P1 pair and determine the 'matching' field $B_{\mathrm{m}}$ (where the P1 Zeeman splitting coincides with the NV $\left|m_{S}=0\right\rangle \leftrightarrow$ $\left|m_{S}=-1\right\rangle$ energy difference) as a function of the polar angle (Fig. 4c). Below $\theta \sim 40$ deg., $B_{\mathrm{m}}$ varies over a moderate range $(50-90 \mathrm{mT})$ thus making it possible to envision polarization transfer over a sizable polar cone of relative crystal orientations with only modest practical means. Recent experimental observations demonstrating efficient P1-mediated carbon polarization in diamond for $\theta$ approaching $\sim 20$ deg. are consistent with this notion ${ }^{17}$.

Fig. 4d shows a polar representation of the proton polarization as a function of the angular coordinates $\theta$ and $\phi$. We find a mild dependence on the azimuthal angle, but the polar response exhibits sharp minima superimposed to an overall decay. The latter stem from quantum interference between consecutive LZ crossings (within the same sweep) and their angular positions depend on the considered couplings and exact conditions of the sweep (see insert to Fig. 4d). The overall envelope, on the other 
(a)

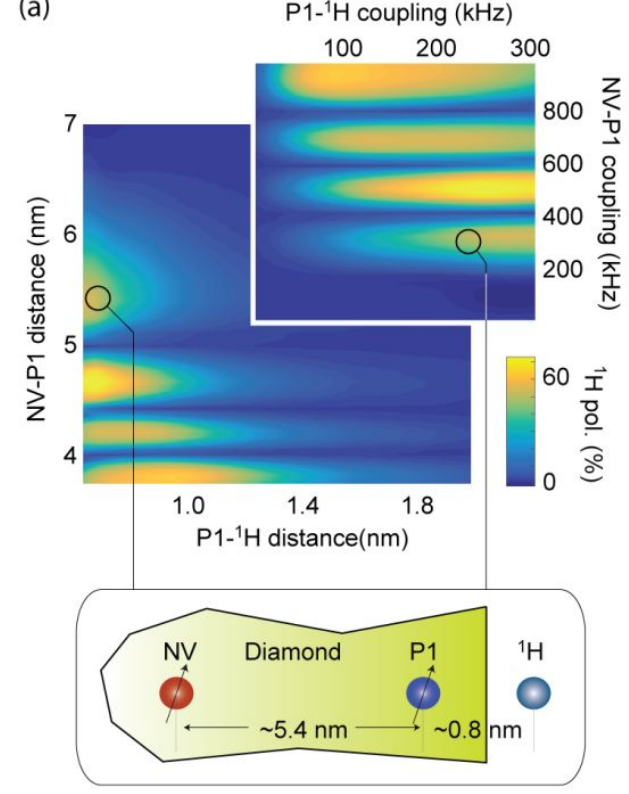

(b)
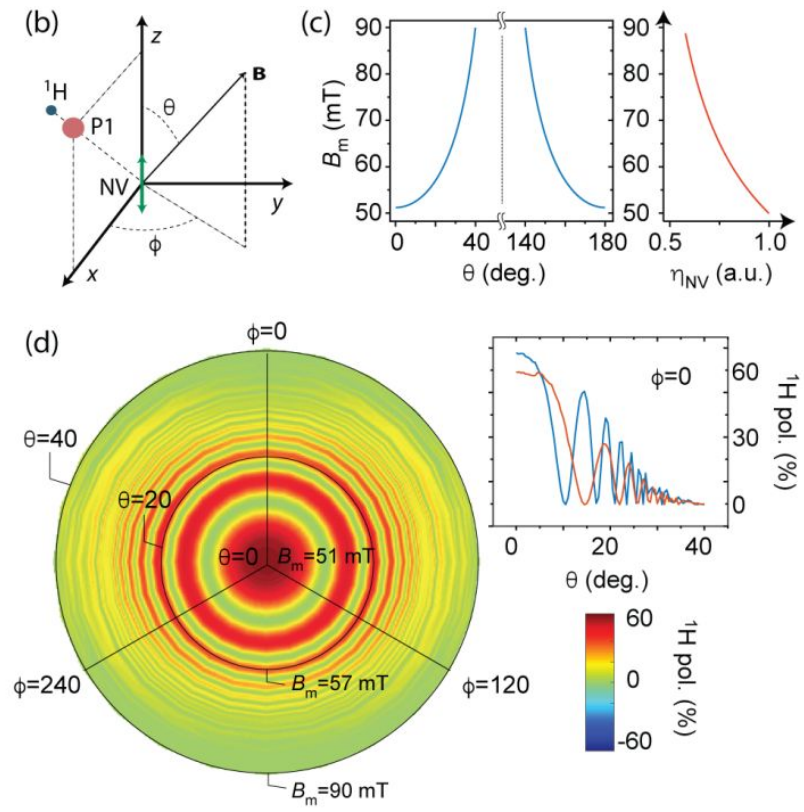

Figure 4. The impact of spin coupling heterogeneity and orientation disorder. (a) Proton polarization as a function of the NV-P1 and $\mathrm{P} 1-{ }^{1} \mathrm{H}$ distances. The upper insert shows the same plot as a function of the corresponding coupling strenghts. Efficient DNP can be attained for NV-P1 (P1-1 H) couplings down to $350 \mathrm{kHz}(250 \mathrm{kHz})$ corresponding to spin distances of $\sim 5.4 \mathrm{~nm}(\sim 0.8 \mathrm{~nm})$, as sketched in the lower insert. (b) Schematics of the reference frame for the case where the $\mathbf{B}$ field and the NV symmetry axis are not collinear. For simplicity, we choose the location of the proton along the axis connecting the NV and P1 spins. (c) Matching field $B_{\mathrm{m}}$ as a function of the polar angle $\theta$. The side graph shows the NV optical pumping efficiency $\eta_{\mathrm{NV}}$ as a function of $B_{\mathrm{m}}$. (d) Polar representation of the ${ }^{1} \mathrm{H}$ polarization as a function of the magnetic field orientation assuming $\beta_{\text {up }}=3.25 \mathrm{mT} / \mathrm{ms}$ and $\beta_{\text {down }}=20 \mathrm{mT} / \mathrm{ms}$; the plot takes into account the NV spin pumping efficiency $\eta_{\mathrm{NV}} \leq 1$ at a given field $B_{\mathrm{m}}$. The blue trace in the upper right insert shows the cross section in the main plot for $\phi=0$; the red trace provides the same information but for $\beta_{\text {up }}=6 \mathrm{mT} / \mathrm{ms}$ and $\beta_{\text {down }}=10 \mathrm{mT} / \mathrm{ms}$. In (a) we assume $\theta=0$, and in (d) we make the NV-P1 $\left(\mathrm{P} 1-{ }^{1} \mathrm{H}\right)$ coupling equal to $500 \mathrm{kHz}(100 \mathrm{kHz})$. The total number of DNP cycles at each point is $n=56$; all other conditions as in Fig. 2a.

hand, arises partly from modest NV spin initialization $\eta_{\mathrm{NV}}$ at higher matching fields $B_{\mathrm{m}}$ (right plot in Fig. 4c) combined with poor polarization transfer efficiency. We therefore conclude this form of proxy-spin mediated DNP is confined to the solid cone defined by $\theta \sim 20 \mathrm{deg}$., which, for the case of a powdered diamond sample, corresponds to limiting the field sweep to the range $51-57 \mathrm{mT}$. This robustness to field misalignment (or, by the same token, field heterogeneity) is in strong contrast with prior demonstrations of P1-assisted $\mathrm{DNP}^{17,18}$, where contributions from all crystallites - positive or negative depending on the local field strength and/or relative orientation - average out. This problem is particularly acute for weakly coupled nuclei because they polarize (positively or negatively) only in a close vicinity of the matching field ${ }^{17,18,30}$.

Before concluding, we note that in a realistic environment, the NV may simultaneously interact with P1s other than the near-surface proxy we modeled, thus prompting the question as to whether the flow of polarization can be diverted off the target. To investigate this possibility, we consider an extension of the case in Fig. $4 \mathrm{a}$, where the spin cluster is modified to include an additional 'bystander' P1 strongly coupled to the NV but far from the target nucleus. Remarkably, we find the ${ }^{1} \mathrm{H}$ spin can still polarize efficiently to about $50 \%$ of the ideal value, provided the effective bystander spin recycling time $T_{1}^{\text {B1) }}$ (defined by spin-lattice relaxation or spin diffusion) satisfies $\tau_{\mathrm{LZ}}<T_{1}^{(\mathrm{B} 1)} \lesssim T_{\mathrm{c}} / 2 \ll T_{1}^{(1 \mathrm{H})}$, with $T_{1}^{(1 \mathrm{H})}$ denoting the ${ }^{1} \mathrm{H}$ nuclear spin-lattice relaxation time. Since the starting distance between the NV and proxy spin is $5 \mathrm{~nm}$, the presence of an additional bystander P1 at $\sim 3.6 \mathrm{~nm}$ from the NV is only likely at P1 concentration of $\sim 50 \mathrm{ppm}$ or greater, meaning that the technique is expected to work reasonably well in representative diamond surfaces. We refer the reader to the Supplementary Information, Section VII, for further details on these calculations.

In summary, cross-relaxation of the NV center spin with surrounding paramagnetic impurities at low magnetic fields can be exploited to induce spin polarization of nuclear targets not interacting with the NV. Central to this approach is the Landau-Zener dynamics induced by partly non-adiabatic magnetic field sweeps across the set of avoided crossings from nearly-matched energy differences of the individual NV and P1 spins. Somewhat counterintuitively, our work shows that successive field sweeps in opposite directions contribute constructively to the DNP process, to ultimately yield a net nuclear 
polarization comparable to that of the NV spin, with a sign defined by the relative timing of the optical excitation. This proxy-mediated DNP mechanism does not require the use of microwave, should operate under ambient conditions, and is robust to spin coupling heterogeneity and $\mathrm{NV}$ orientation disorder. Unlike prior demonstrations of P1-assisted NV-DNP, these traits make this approach applicable to diamond powders, and insensitive to magnetic field heterogeneity (both spatial and temporal), or system fluctuations (induced, e.g., by laser heating). Interestingly, our calculations indicate that spin-lattice relaxation of the proxy spin can have a positive impact on the DNP efficiency, either by broadening the range of sweep velocities where the transfer remains efficient, and/or by mitigating the adverse effect of strong decoherence (i.e., the regime where $T_{2}^{(\mathrm{P} 1)} \ll \tau_{\mathrm{LZ}}$ ). A table with a summary on the range of conditions where this technique is expected to work well can be found in the Supplementary Information, Section VIII.

Because the present technique promises to remain effective even for weak spin couplings, we anticipate proxy-mediated DNP can transfer polarization directly to nuclear spin targets outside the diamond crystal. We contrast this mechanism to nuclear-spin-diffusion transfer, inherently slower and thus more sensitive to shallowdefect-induced spin-lattice relaxation. Finally, we anticipate several extensions of the present technique, for example, in the form of double-resonance schemes at low magnetic fields (e.g., $\sim 10 \mathrm{mT}$ ) designed to recreate analogous three-spin-LZ-dynamics in the rotating frame. Potential advantages include the ability to access all NV orientations without compromising on the NV spin pumping efficiency, and the option to separately optimize the sweep velocity and repetition rates via the use of frequency combs ${ }^{31}$.

\section{ASSOCIATED CONTENT}

Supporting Information: Contains information on the Hamiltonian model, the dynamics of single sweeps, the effect of transversal and longitudinal relaxation, and the global dynamics of multiple cycles by means of a transfer matrix approach.

\section{AUTHOR INFORMATION}

\section{Corresponding author:}

${ }_{\dagger}$ E-mail: cmeriles@ccny.cuny.edu

\section{Notes}

The authors declare no competing financial interests.

\section{ACKNOWLEDGMENTS}

P.R.Z., J.H., D.P. and C.A.M. acknowledge support from the National Science Foundation through grants NSF-1619896 and NSF-1401632, and from Research
Corporation for Science Advancement through a FRED Award; they also acknowledge access to the facilities and research infrastructure of the NSF CREST IDEALS, grant number NSF-HRD-1547830. J.H. acknowledges support from CREST-PRF NSF-HRD 1827037. All authors acknowledge the CUNY High Performance Computing Center (HPCC). The CUNY HPCC is operated by the College of Staten Island and funded, in part, by grants from the City of New York, State of New York, CUNY Research Foundation, and National Science Foundation Grants CNS-0958379, CNS-0855217 and ACI 1126113.

\section{REFERENCES}

1 Atsarkin, V.A. "Dynamic nuclear polarization: Yesterday, today, and tomorrow", J. Phys. Conf. Ser. 2011, 324, 012003.

2 Abrams, D.; Trusheim, M.E.; Englund, D.; Shattuck, M.D.; Meriles, C.A. "Dynamic nuclear spin polarization of liquids and gases in contact with nanostructured diamond", Nano Lett. 2014, 14, 2471-2478.

${ }^{3}$ King, J.P.; Jeong, K.; Vassiliou, C.C.; Shin, C.S.; Page, R.H.; Avalos, C.E.; Wang, H-J.; Pines, A. "Room-temperature in situ nuclear spin hyperpolarization from optically pumped nitrogen vacancy centres in diamond", Nat. Commun. 2015, 6, 8965.

${ }^{4}$ London, P.; Scheuer, J.; Cai, J.-M.; Schwarz, I.; Retzker, A.; Plenio, M.B.; Katagiri, M.; Teraji, T.; Koizumi, S.; Isoya, J.; Fischer, R.; McGuinness, L.P.; Naydenov, B.; Jelezko, F. "Detecting and polarizing nuclear spins with double resonance on a single electron spin", Phys. Rev. Lett. 2013, 111, 067601.

5 Fischer, R.; Bretschneider, C.O; London, P.; Budker, D; Gershoni, D.; Frydman, L. "Bulk Nuclear Polarization Enhanced at Room Temperature by Optical Pumping", Phys. Rev. Lett. 2013, 111, 057601.

${ }^{6}$ Green, B.L.; Breeze, B.G.; Rees, G.J.; Hanna, J.V.; Chou, J.P.; Ivády, V.; Gali, A.; Newton, M.E. "All-optical hyperpolarization of electron and nuclear spins in diamond", Phys. Rev. B 2017, 96, 054101.

${ }^{7}$ Falk, A.L.; Klimov, P.V.; Ivády, V.; Szász, K.; Christle, D.J.; Koehl, W.F.; Gali, Á.; Awschalom, D.D. "Optical polarization of nuclear spins in silicon carbide", Phys. Rev. Lett. 2015, 114, 247603.

${ }^{8}$ Waddington, D.E.J.; Sarracanie, M.; Zhang, H.; Salameh, N.; Glenn, D.R.; Rej, E.; Gaebel, T.; Boele, T.; Walsworth, R.L.; Reilly, D.J.; Rosen, M.S. "Nanodiamond-enhanced MRI via in situ hyperpolarization", Nat. Commun. 2017, 8, 15118.

${ }^{9}$ Aptekar, J.W.; Cassidy, M.C.; Johnson, A.C.; Barton, R.A.; Lee, M.; Ogier, A.C.; Vo, C.; Anahtar, M.N.; Ren, Y.; Bhatia, S.N.; Ramanathan, C.; Cory, D.G.; Hill, A.L.; Mair, R.W.; Rosen, M.S.; Walsworth, R.L.; Marcus, C.M. "Silicon nanoparticles as hyperpolarized magnetic resonance imaging agents", ACS Nano 2009, 3, 4003-4008.

${ }^{10}$ Broadway, D.A.; Tetienne, J-P.; Stacey, A.; Wood, J.D.A.; Simpson, D.A.; Hall, L.T.; Hollenberg, L.C.L. "Quantum probe hyperpolarisation of molecular nuclear spins", Nat. Commun. 2018, 9, 1246.

${ }^{11}$ Shagieva, F.; Zaiser, S.; Neumann, P.; Dasari, D.B.R.; Stöhr, R.; Denisenko, A.; Reuter, R.; Meriles, C.A.; Wrachtrup, J. 
"Microwave-assisted cross-polarization of nuclear spin ensembles from optically-pumped nitrogen-vacancy centers in diamond", Nano Lett. 2018, 18, 3731-3737.

12 Tateishi, K.; Negoro, M.; Nishida, S.; Kagawa, A.; Morita, Y.; Kitagawa, M. "Room temperature hyperpolarization of nuclear spins in bulk", Proc. Natl. Acad. Sci. USA 2014, 111, 75277530.

13 Scott, E.; Drake, M.; Reimer, J.A. "The phenomenology of optically pumped ${ }^{13} \mathrm{C}$ hyperpolarization in diamond at $7.05 \mathrm{~T}$ : Room temperature polarization, orientation dependence, and the effect of defect concentration on polarization dynamics", $J$. Mag. Reson. 2016, 264, 154.

14 Ajoy, A.; Liu, K.; Nazaryan, R.; Lv, X.; Zangara, P.R.; Safvati, B.; Wang, G.; Arnold, D.; Li, G.; Lin, A.; Raghavan, P.; Druga, E.; Dhomkar, S.; Pagliero, D.; Reimer, J.A.; Suter, D.; Meriles, C.A.; Pines, A. "Orientation-independent roomtemperature optical ${ }^{13} \mathrm{C}$ hyperpolarization in powdered diamond", Science Adv. 2018, 4, eaar5492.

15 Zangara, P.R.; Dhomkar, S.; Ajoy, A.; Liu, K.; Nazarian, R.; Pagliero, D.; Suter, D.; Reimer, J.A.; Pines, A.; Meriles, C.A. "Dynamics of frequency-swept nuclear spin optical pumping in powdered diamond at low magnetic fields", Proc. Natl. Acad. Sci. USA 2019, 116, 2512-2520.

16 Thurber, K.R.; Tycko, R. "Theory for cross effect dynamic nuclear polarization under magic-angle spinning in solid state nuclear magnetic resonance: the importance of level crossings". J. Chem. Phys. 2012, 137, 084508.

17 Pagliero, D.; Koteswara Rao, K.R.; Zangara, P.R.; Dhomkar, S.; Wong, H.H.; Abril, A.; Aslam, N.; Parker, A.; King, J.; Avalos, C.E.; Ajoy, A.; Wrachtrup, J.; Pines, A.; Meriles, C.A. "Multispin-assisted optical pumping of bulk 13C nuclear spin polarization in diamond", Phys. Rev. B 2018, 97, 024422.

18 Wunderlich, R.; Kohlrautz, J.; Abel, B.; Haase, J.; Meijer, J. "Optically induced cross relaxation via nitrogen-related defects for bulk diamond ${ }^{13} \mathrm{C}$ hyperpolarization", Phys. Rev. B 2017, 96, 220407(R).

19 Schaffry, M.; Gauger, E.M.; Morton, J.J.L.; Benjamin, S.C. "Proposed spin amplification for magnetic sensors employing crystal defects", Phys. Rev. Lett. 2011, 107, 207210.

${ }^{20}$ Laraoui, A.; Hodges, J.S; Meriles, C.A. "Nitrogen-Vacancyassisted magnetometry of paramagnetic centers in an individual diamond nanocrystal", Nano Lett. 2012, 12, 3477-3482.

${ }^{21}$ Cappellaro, P.; Goldstein, G.; Hodges, J.S.; Jiang, L.; Maze, J.R.; Sørensen, A.S.; Lukin, M.D. "Environment-assisted metrology with spin qubits", Phys. Rev. A 2012, 85, 032336.

${ }^{22}$ Hauf, M.V.; Grotz, B.; Naydenov, B.; Dankerl, M.; Pezzagna, S.; Meijer, J.; Jelezko, F.; Wrachtrup, J.; Stutzmann, M.; Reinhard, F.; Garrido, J.A. "Chemical control of the charge state of nitrogen-vacancy centers in diamond", Phys. Rev. B 2011, 83, 081304(R).

23 Armstrong, S.; Rogers, L.J.; McMurtrie, R.L.; Manson, N.B. "NV-NV electron-electron spin and NV-NS electron - electron and electron-nuclear spin interaction in diamond", Phys. Proc. 2010, 3, 1569.

24 Grossert, C.; Leder, M.; Denisov, S.; Hänggi, P.; Weitz, M. "Experimental control of transport resonances in a coherent quantum rocking ratchet", Nat. Commun. 2016, 7, 10440.

25 Shevchenko, S.N.; Ashhab, S.; Nori, F. "Landau-ZenerStückelberg interferometry", Phys. Rep. 2010, 492, 1

${ }^{26}$ Kayanuma, Y. "Phase coherence and nonadiabatic transition at a level crossing in a periodically driven two-level system", Phys. Rev. B 1993, 47, 9940.

27 Kayanuma, Y. "Population Inversion in Optical Adiabatic Rapid Passage with Phase Relaxation", Phys. Rev. Lett. 1987, 58, 1934.

${ }^{28}$ Ao, P; Rammer, J. "Quantum dynamics of a two-state system in a dissipative environment", Phys. Rev. B 1991, 43, 5397.

29 Staudacher, T.M.; Raatz, N.; Pezzagna, S.; Meijer, J.; Reinhard, F.; Meriles, C.A.; Wrachtrup, J. "Probing molecular dynamics at the nanoscale via an individual paramagnetic center", Nature Commun. 2015, 6, 8527.

30 Wood, J.D.A.; Tetienne, J-P.; Broadway, D.A.; Hall, L.T.; Simpson, D.A.; Stacey, A.; Hollenberg, L.C.L. "Microwave-free nuclear magnetic resonance at molecular scales", Nat. Commun. 2017, 8, 15950 .

31 Ajoy, A.; Nazaryan, R.; Liu, K.; Lv, X.; Safvati, B.; Wang, G.; Druga, E.; Reimer, J.A.; Suter, D.; Ramanathan, C.; Meriles, C.A.; Pines, A. "Enhanced dynamic nuclear polarization via swept microwave frequency combs", Proc. Natl. Acad. Sci. USA 2018, 115, 10576. 
(a)

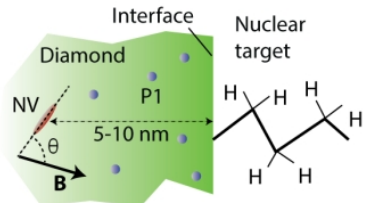

(b)

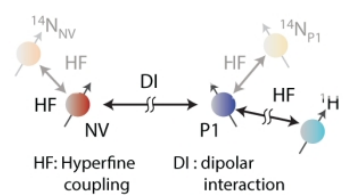

(c)

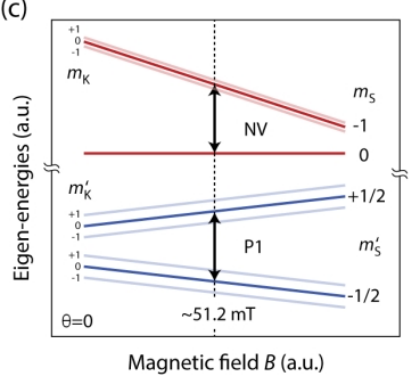

(e)
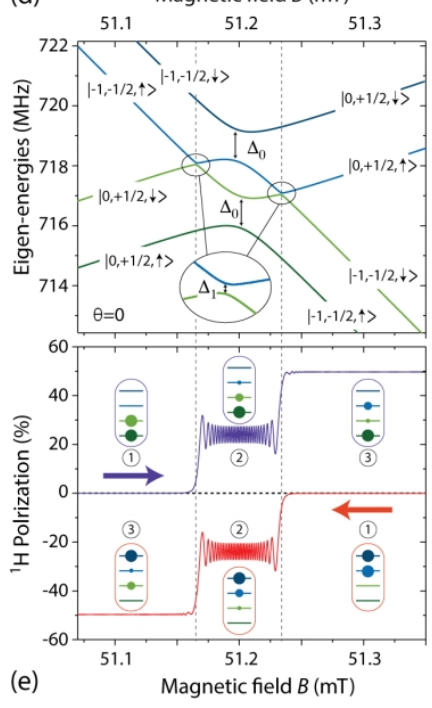

(f)

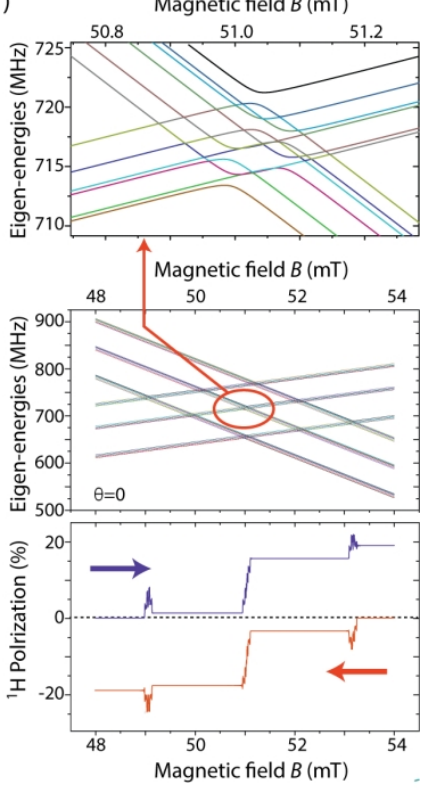

$165 \times 106 \mathrm{~mm}(300 \times 300 \mathrm{DPI})$

30

31

32

33

34

35

36

37

38

39

40

41

42

43

44

45

46

47

48

49

50

51

52

53

54

55

56

57

58

59

60

ACS Paragon Plus Environment 
(a)

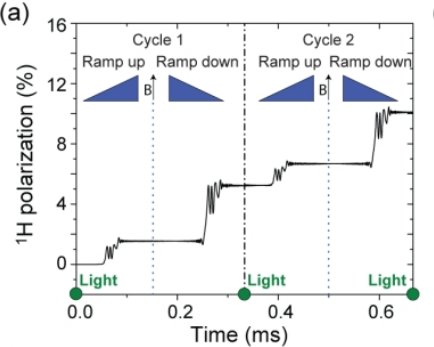

(b)

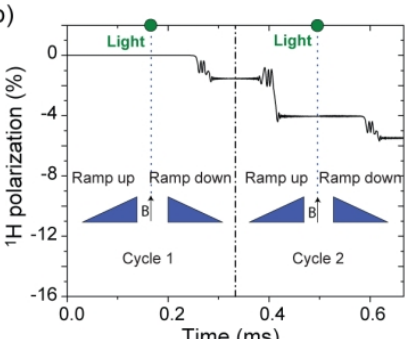

(d)

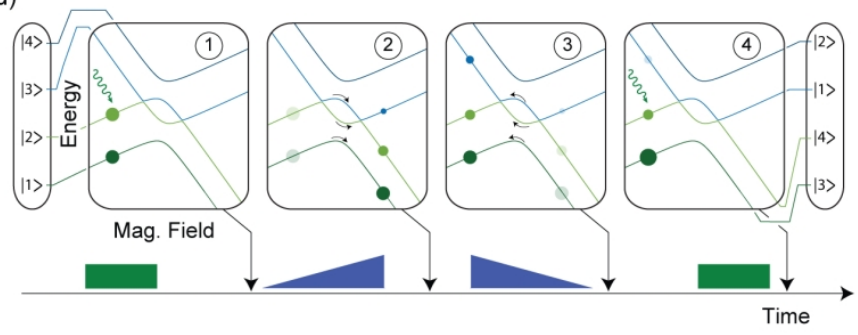

$162 \times 89 \mathrm{~mm}(300 \times 300 \mathrm{DPI})$

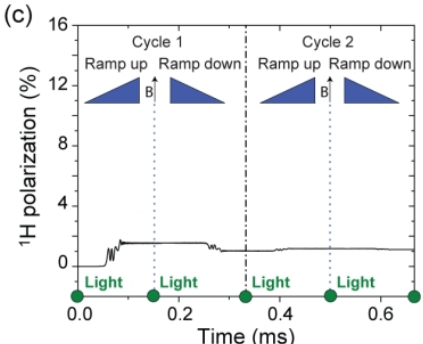

(e)

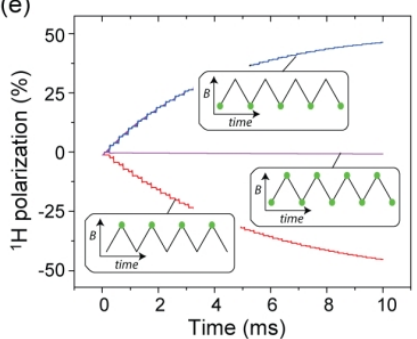



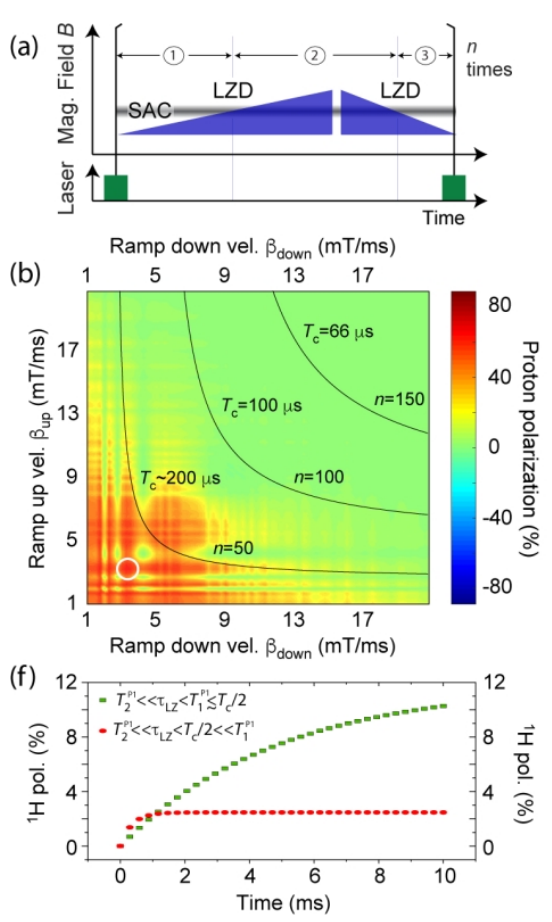

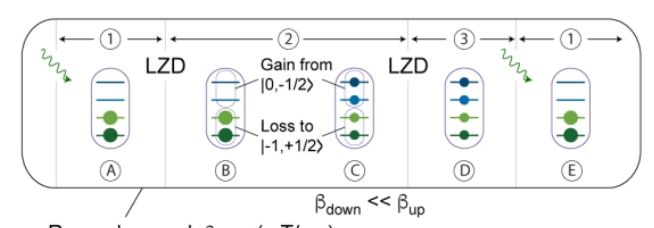

(c) 1

Ramp down vel. $\beta_{\text {down }}$ (mT/ms)

Ramp down vel. $\beta_{\text {down }}(\mathrm{mT} / \mathrm{ms})$

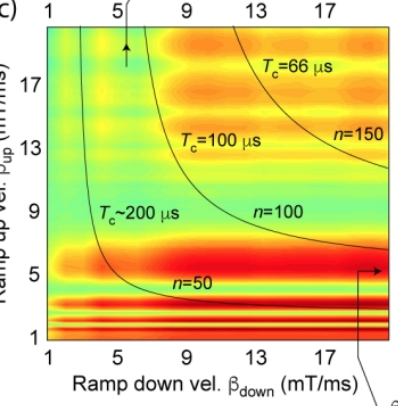

$\begin{array}{lllll}1 & 5 & 9 & 13 & 17\end{array}$
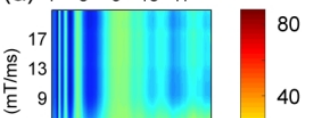

है
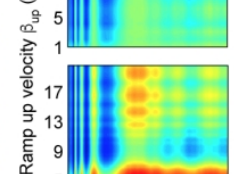

(e) $\begin{array}{lllll}1 & 5 & 9 & 13 & 17\end{array}$

(e) Ramp down vel. $\beta_{\text {down }}(\mathrm{mT} / \mathrm{ms})$
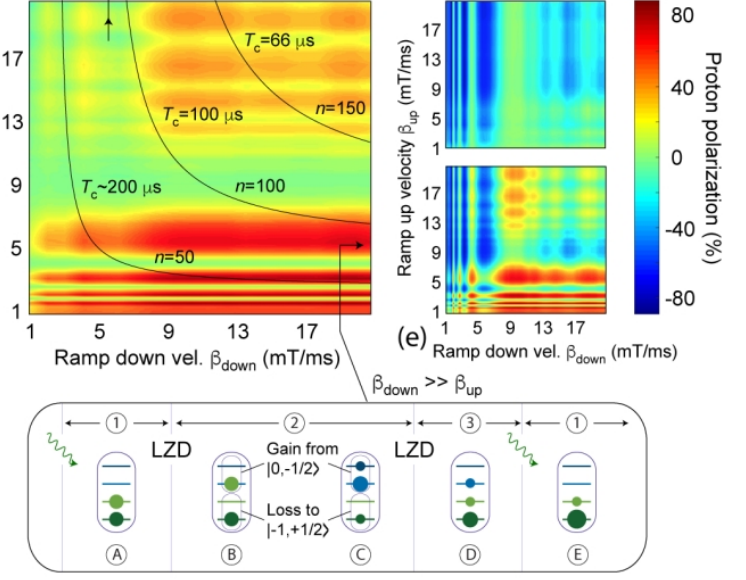

$153 \times 105 \mathrm{~mm}(300 \times 300 \mathrm{DPI})$ 
(a)

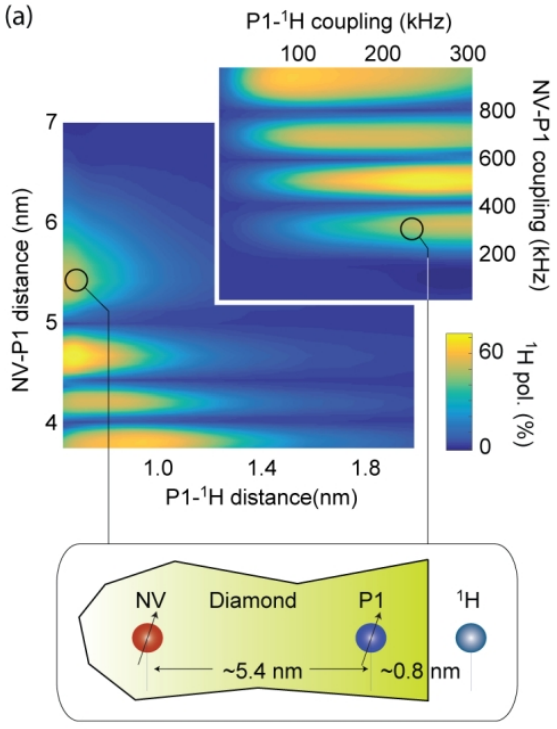

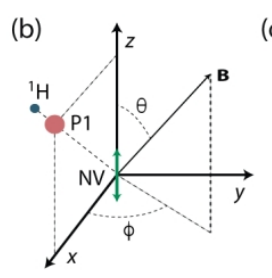
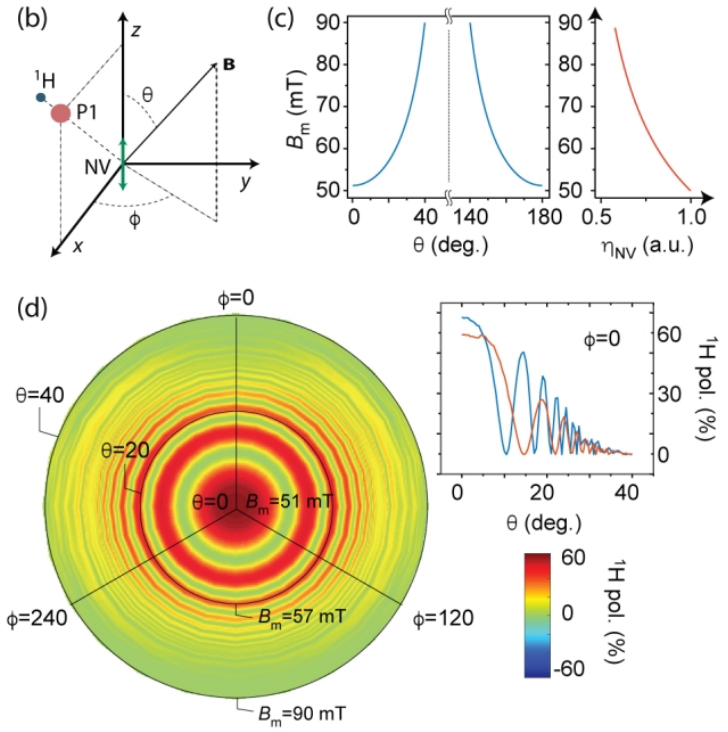

$146 \times 81 \mathrm{~mm}(300 \times 300 \mathrm{DPI})$ 


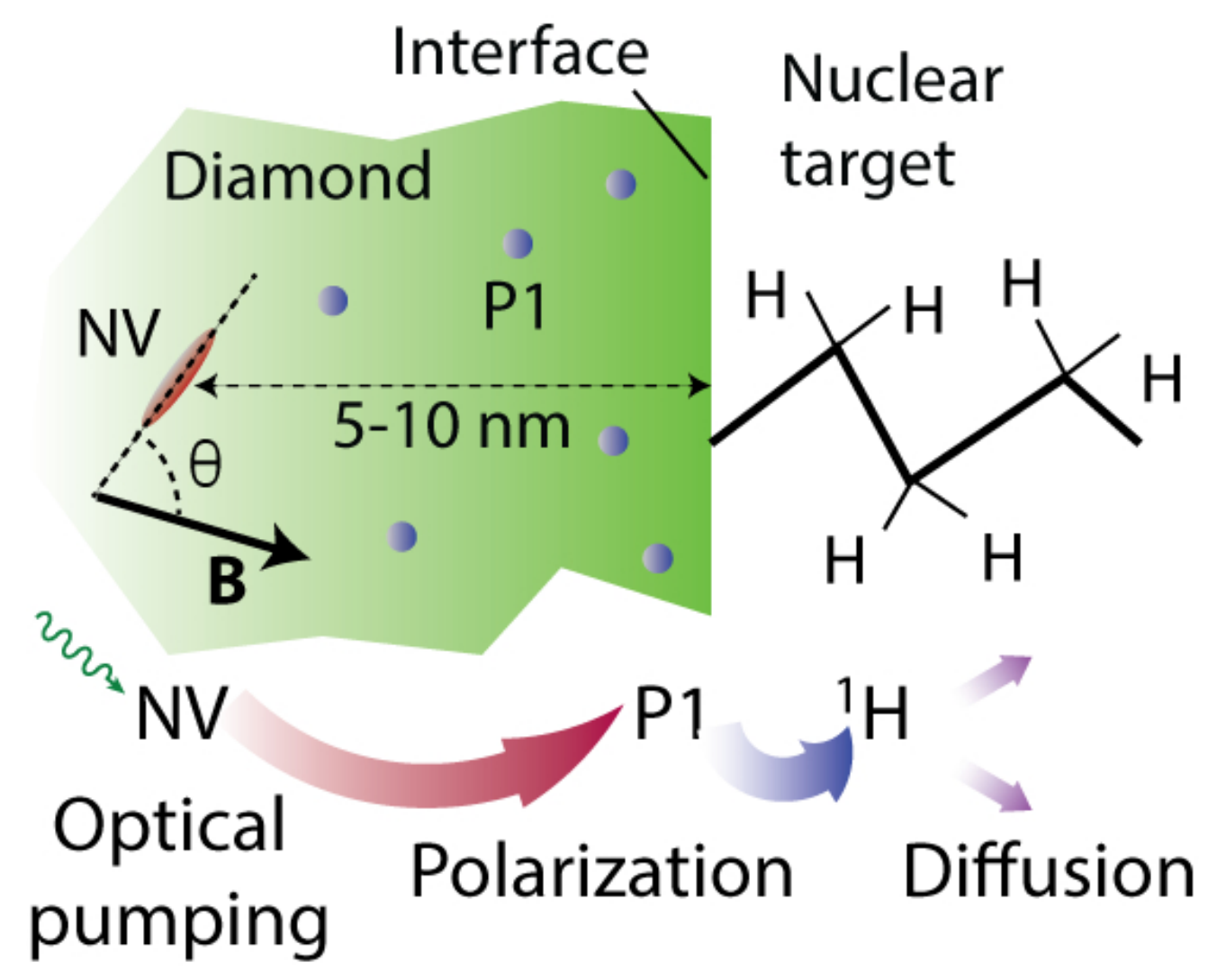

$47 \times 38 \mathrm{~mm}(300 \times 300 \mathrm{DPI})$ 